CREAT. MATH. INFORM.

Volume 31 (2022), No. 1,

Pages $43-49$

\title{
On extensions of pseudo-valuations on BCK algebras
}

\author{
Dumitru Busneag, Dana Piciu and Mihaela Istrata
}

\begin{abstract}
In this paper we define a pseudo-valuation on a BCK algebra $(A, \rightarrow, 1)$ as a real-valued function $v: A \rightarrow \mathbf{R}$ satisfying $v(1)=0$ and $v(x \rightarrow y) \geq v(y)-v(x)$, for every $x, y \in A ; v$ is called a valuation if $x=1$ whenever $v(x)=0$. We prove that every pseudo-valuation (valuation) $v$ induces a pseudo-metric (metric) on $A$ defined by $d_{v}(x, y)=v(x \rightarrow y)+v(y \rightarrow x)$ for every $x, y \in A$, where $\rightarrow$ is uniformly continuous in both variables. The aim of this paper is to provide several theorems on extensions of pseudo-valuations (valuations) on BCK algebras.
\end{abstract}

\section{INTRODUCTION AND BASIC RESULTS}

$\mathrm{BCK}$ algebras are an important class of logical algebras investigated by many researchers (see [2], [3], [6], [8], [9], [10], [13]). BCK algebras were originally introduced by Isèki in [9]. Further properties of them and their connections with other fuzzy structures were established by Iorgulescu in [8].

In [1], Busneag defined pseudo-valuations on Hilbert algebras and proved that every pseudo-valuation induced a pseudo-metric. Using this model, in [4], [5], [7], [11], [12], [14], [15], [16] is introduced the notion of pseudo-valuation on BCK, BCI, BCC algebras and several properties are discussed.

The main goal of this paper is to introduce the notions of pseudo-valuation and valuation on BCK algebras and to prove theorems on extensions of pseudo-valuations (valuations) on BCK algebras.

The paper is organized as follows: In Section 1 we review some relevant concepts relative to BCK algebras. In Section 2 we introduce the notions of pseudo-valuation and valuation on BCK algebras and we induce a pseudo-metric by using pseudo-valuations on BCK algebras (Theorem 2.1). Also, we show that the binary operation $\rightarrow$ is uniformly continuous, see Corollary 2.1. Finally, we prove some theorems (2.2 and 2.3) on extensions of pseudo-valuations (valuations) on BCK algebras. Section 3 contains results about pseudo-valuations on the dual BCK algebra, see Theorem 3.4 and the final section contains conclusions, open problems and future work about the presented topics.

A BCK algebra is an algebra $(A, \rightarrow, 1)$ of type $(2,0)$ satisfying:

$\left(a_{1}\right) x \rightarrow x=1$;

$\left(a_{2}\right)$ If $x \rightarrow y=y \rightarrow x=1$, then $x=y$;

(B) $x \rightarrow y \leq(y \rightarrow z) \rightarrow(x \rightarrow z)$;

(C) $x \rightarrow(y \rightarrow z)=y \rightarrow(x \rightarrow z)$;

$(K) x \leq y \rightarrow x$.

Example 1.1. ([8]) We give an example of a finite bounded BCK algebra. Let $A=\{0, a, b, c, 1\}$ with $0<a, b<c<1$, but $a, b$ are incomparable. $A$ becomes a BCK algebra relative to the

Received: 08.01.2021. In revised form: 18.11.2021. Accepted: 25.11.2021

2010 Mathematics Subject Classification. 06F35, 03G25, 18A15, 18 C05.

Key words and phrases. BCK algebra, pseudo-valuation, valuation.

Corresponding author: Dana Piciu; piciudanamarina@yahoo.com 
following operation:

\begin{tabular}{c|ccccc}
$\rightarrow$ & 0 & $a$ & $b$ & $c$ & 1 \\
\hline 0 & 1 & 1 & 1 & 1 & 1 \\
$a$ & $b$ & 1 & $b$ & 1 & 1 \\
$b$ & $a$ & $a$ & 1 & 1 & 1 \\
$c$ & 0 & $a$ & $b$ & 1 & 1 \\
1 & 0 & $a$ & $b$ & $c$ & 1
\end{tabular}

If $A$ is a BCK algebra, then the relation $\leq$ defined by $x \leq y$ iff $x \rightarrow y=1$ is a partial order on $A$ (called the natural order); with respect to this order 1 is the largest element of $A$. A bounded $B C K$ algebra is a BCK algebra with a smallest element 0 relative to the natural order. For a BCK algebra $A$, two elements $x, y \in A$ and a natural number $n \geq 1$ we denote $x \rightarrow_{n} y=x \rightarrow(x \rightarrow \ldots(x \rightarrow y) \ldots)$, where $n$ indicates the number of occurrences of $x$.

In BCK algebras we have the following rules of calculus (see [2] and [10]):

$\left(c_{1}\right) x \rightarrow 1=1,1 \rightarrow x=x, x \leq(x \rightarrow y) \rightarrow y, x \rightarrow y \leq(z \rightarrow x) \rightarrow(z \rightarrow y)$;

$\left(c_{2}\right)$ If $x \leq y$, then for every $z \in A, z \rightarrow x \leq z \rightarrow y$ and $y \rightarrow z \leq x \rightarrow z$.

For a BCK algebra $A$ and $x_{1}, \ldots, x_{n}, x \in A(n \geq 1)$ we define $\left(x_{1}, \ldots, x_{n} ; x\right)=x_{1} \rightarrow$ $\left(x_{2} \rightarrow \ldots\left(x_{n} \rightarrow x\right) \ldots\right)$. If $\sigma$ is a permutation of $\{1, \ldots, n-1\}, n \geq 2$, then:

$\left(c_{3}\right)\left(x_{\sigma(1)}, \ldots, x_{\sigma(n-1)} ; x_{n}\right)=\left(x_{1}, \ldots, x_{n-1} ; x_{n}\right)$;

$\left(c_{4}\right) x \rightarrow\left(x_{1}, \ldots, x_{n-1} ; x_{n}\right)=\left(x, x_{1}, \ldots, x_{n-1} ; x_{n}\right)$;

Let $A$ be a BCK algebra. A subset $D$ of $A$ is called a deductive system of $A$ if $1 \in D$ and $x, x \rightarrow y \in D$ implies $y \in D$.

Clearly, if $D$ is a deductive system of $A$ and $x \leq y$ with $x \in D$, then $y \in D$.

We denote by $D s(A)$ the set of all deductive systems of a BCK algebra $A$.

For a non-empty subset $X \subseteq A$, we denote by $<X>=\cap\{D \in D s(A): X \subseteq D\}$; $<X>$ is called the deductive system of $A$ generated by $X$. If $X=\left\{x_{1}, \ldots, x_{n}\right\}$ we denote $<$ $\left\{x_{1}, \ldots, x_{n}\right\}>$ by $<x_{1}, \ldots, x_{n}>$; also, we denote by $<a>$ the deductive system generated by $\{a\}$. It is easy to prove (see [2]) that $\langle a\rangle=\left\{x \in A: a \rightarrow_{n} x=1\right.$, for some natural number $n \geq 1\}(<a>$ is called principal).

Let $A$ be a bounded BCK algebra. An element $x \in A$ is called boolean (see [6]) if $\langle x\rangle$ $\left.\cap<x^{*}\right\rangle=\{1\}$. Let $B(A)$ the set of all boolean elements of $A$.

In [2] it is proved that if $A$ is a BCK algebra and $X \subseteq A$ then

$\left(c_{5}\right)<X>=\left\{x \in A:\left(x_{1}, \ldots, x_{n} ; x\right)=1\right.$, for some $x_{1}, \ldots, x_{n} \in X$ and $\left.n \geq 1\right\}$;

$\left(c_{6}\right)$ If $D_{1}, D_{2} \in D s(A)$ and we define $D_{1} \vee D_{2}=<D_{1} \cup D_{2}>$, then $D_{1} \vee D_{2}=\{x \in A$ : $d_{1} \rightarrow\left(d_{2} \rightarrow x\right)=1$, for some $d_{1} \in D_{1}$ and $\left.d_{2} \in D_{2}\right\}$.

Remark 1.1. If $D \in D s(A)$, then $D$ is a BCK subalgebra of $A$ (since $1 \in D$ and if $x, y \in D$ from $y \leq x \rightarrow y$ we deduce that $x \rightarrow y \in D$ ).

\section{Pseudo-VAluations (VAluations) on BCK Algebras}

Using the model of Hilbert algebras (see [1]), in this section we introduce the notions of pseudo-valuations and valuations on BCK algebras and we prove some theorems of extension for these.

Let $A$ be a BCK algebra. A real-valued function $v: A \rightarrow \mathbf{R}$ is called a pseudo-valuation on $A$ if $v(1)=0$ and $\left(^{*}\right): v(x \rightarrow y) \geq v(y)-v(x)$, for every $x, y \in A$. The pseudo-valuation $v$ is called valuation if $v(x)=0$ implies $x=1$.

If we interpret $A$ as an implicational calculus, $x \rightarrow y$ as the proposition $x \Rightarrow y$ and 1 as truth, a pseudo-valuation on $A$ can be interpreted as a "falsity-valuation".

Example 2.2. $v: A \rightarrow \mathbf{R}, v(x)=0$ for every $x \in A$ is a pseudo-valuation on $A$. 
Example 2.3. If $D \in D s(A)$ and $0 \leq r \in \mathbf{R}$, then $v_{D}: A \rightarrow \mathbf{R}, v_{D}(x)=0$, if $x \in D$ and $r$ otherwise, is a pseudo-valuation on $A$. Indeed, $v_{D}(1)=0$ since $1 \in D$. Let $x, y \in A$. If $x, y \in D$, since $y \leq x \rightarrow y$ we obtain $x \rightarrow y \in D$. So, $v_{D}(x \rightarrow y)=v_{D}(x)=v_{D}(y)=0$ and $v_{D}(x \rightarrow y)=v_{D}(y)-v_{D}(x)$. If $x, y \notin D$, then $v_{D}(y)-v_{D}(x)=r-r=0 \leq v_{D}(x \rightarrow y)$. If $x \notin$ $D$ and $y \in D$ we deduce that $x \rightarrow y \in D$, so $0=v_{D}(x \rightarrow y) \geq v_{D}(y)-v_{D}(x)=0-r=-r$. If $y \notin D$ and $x \in D$ then $x \rightarrow y \notin D$. We obtain $r=v_{D}(x \rightarrow y)=v_{D}(y)-v_{D}(x)=r-0=r$.

Remark 2.2. Let $A$ be a non trivial BCK algebra, $D \in D s(A), r \geq 0$ and $v_{D}: A \rightarrow \mathbf{R}$ the function given by $v_{D}(x)=0$ if $x \in D$ and $r$ otherwise. Then $v_{D}$ is a valuation if and only if $D=\{1\}$ and $r>0$.

Example 2.4. Let $M$ be a finite set with $n$ elements and $A=P(M)$ be the power set of $M$ (the set of all subsets of $M)$. Then $\left(P(M), \cap, \cup, C_{M}, \varnothing, M\right)$ is a Boolean algebra (where for $\left.X \subseteq M, C_{M}(X)=M \backslash X\right)$. The function $v: P(M) \rightarrow \mathbf{R}$, defined by $v(X)=n-n(X)$ is a valuation on $A$, where $n(X)$ is the number of elements of $X$. Indeed, $v(M)=0$. Let $X, Y \subseteq M$. We have $v(X \rightarrow Y)=v\left(C_{M} X \cup Y\right)=n-n\left(C_{M} X \cup Y\right)=n-n\left(C_{M} X\right)-n(Y)+$ $n\left(C_{M} X \cap Y\right)=n(X)-n(Y)+n\left(C_{M} X \cap Y\right) \geq n(X)-n(Y)=v(Y)-v(X)$. Obviously, $v(X)=0$ iff $X=M$.

Lemma 2.1. If $v: A \rightarrow \mathbf{R}$ is a pseudo-valuation on $A$ and $x, x_{1}, \ldots, x_{n} \in A$ such that $\left(x_{1}, \ldots, x_{n} ; x\right)$ $=1$ then

$$
\left(c_{7}\right) v(x) \leq \sum_{i=1}^{n} v\left(x_{i}\right)
$$

Proof. $0=v(1)=v\left(\left(x_{1}, \ldots, x_{n} ; x\right)\right) \geq v(x)-\sum_{i=1}^{n} v\left(x_{i}\right)$, so $v(x) \leq \sum_{i=1}^{n} v\left(x_{i}\right)$.

A pseudo-valuation $v: A \rightarrow \mathbf{R}$ is called decreasing if $v(x) \geq v(y)$ for every $x, y \in A$ with $x \leq y$.

Lemma 2.2. A pseudo-valuation $v$ is a positive decreasing function satisfying

$\left(c_{8}\right) v(x \rightarrow y)+v(y \rightarrow z) \geq v(x \rightarrow z)$, for any $x, y, z \in A$.

Proof. If in $\left(^{*}\right)$ we put $y=1$ we obtain $v(x \rightarrow 1) \geq v(1)-v(x)$, so $v(x) \geq 0$, for every $x \in A$. If $x \leq y$, then $x \rightarrow y=1$, so from $\left(^{*}\right)$ we deduce that $0=v(1)=v(x \rightarrow y) \geq v(y)-v(x)$. We conclude that $v(x) \geq v(y)$ for every $x \leq y$, so, $v$ is a decreasing function. Let now $x, y, z \in A$. Since $v$ is a decreasing function, from $(B)$, we deduce that $v(x \rightarrow y) \geq v((y \rightarrow$ $z) \rightarrow(x \rightarrow z)) \geq v(x \rightarrow z)-v(y \rightarrow z)$. Thus, $v(x \rightarrow z) \leq v(x \rightarrow y)+v(y \rightarrow z)$.

We recall that by a pseudo-metric space we mean an ordered pair $(M, d)$, where $M$ is a non-empty set and $d: M \times M \rightarrow \mathbf{R}$ is a positive function satisfying the following properties: $d(x, x)=0, d(x, y)=d(y, x)$ and $d(x, z) \leq d(x, y)+d(y, z)$ for every $x, y, z \in M$. If in the pseudo-metric space $(M, d), d(x, y)=0$ implies $x=y$, then $(M, d)$ is called a metric space.

Theorem 2.1. Let $v: A \rightarrow \mathbf{R}$ be a pseudo-valuation on $A$. If we define $d_{v}: A \times A \rightarrow \mathbf{R}$, $d_{v}(x, y)=v(x \rightarrow y)+v(y \rightarrow x)$, for every $(x, y) \in A \times A$, then

(i) $\left(A, d_{v}\right)$ is a pseudo-metric space satisfying:

$\left(c_{9}\right) \max \left\{d_{v}(x \rightarrow z, y \rightarrow z), d_{v}(z \rightarrow x, z \rightarrow y)\right\} \leq d_{v}(x, y)$, for every $x, y, z \in A$;

(ii) $d_{v}$ is a metric on $A$ iff $v$ is a valuation on $A$.

Proof. $(i)$. Let $x, y, z \in A$. Clearly, $d_{v}(x, y)=d_{v}(y, x) \geq 0$ and $d_{v}(x, x)=v(x \rightarrow x)+$ $v(x \rightarrow x)=v(1)+v(1)=0+0=0$. Also, $d_{v}(x, y)+d_{v}(y, z)=[v(x \rightarrow y)+v(y \rightarrow x)]+$ $[v(y \rightarrow z)+v(z \rightarrow y)]=[v(x \rightarrow y)+v(y \rightarrow z)]+[v(z \rightarrow y)+v(y \rightarrow x)] \stackrel{\left(c_{8}\right)}{\geq} v(x \rightarrow$ 
$z)+v(z \rightarrow x)=d_{v}(x, z)$, hence $d_{v}$ is a pseudo-metric on $A$. Now, we prove $\left(c_{9}\right)$. We have $d_{v}(x \rightarrow z, y \rightarrow z)=v((x \rightarrow z) \rightarrow(y \rightarrow z))+v((y \rightarrow z) \rightarrow(x \rightarrow z))$. Since, from $(B), x \rightarrow y \leq(y \rightarrow z) \rightarrow(x \rightarrow z)$ and $y \rightarrow x \leq(x \rightarrow z) \rightarrow(y \rightarrow z)$ we deduce that $v(x \rightarrow y) \geq v((y \rightarrow z) \rightarrow(x \rightarrow z))$ and $v(y \rightarrow x) \geq v((x \rightarrow z) \rightarrow(y \rightarrow z))$, hence, $d_{v}(x, y)=v(x \rightarrow y)+v(y \rightarrow x) \geq v((y \rightarrow z) \rightarrow(x \rightarrow z))+v((x \rightarrow z) \rightarrow(y \rightarrow z))=$ $d_{v}(x \rightarrow z, y \rightarrow z)$. Since, from $\left(c_{1}\right), x \rightarrow y \leq(z \rightarrow x) \rightarrow(z \rightarrow y)$ and $y \rightarrow x \leq(z \rightarrow$ $y) \rightarrow(z \rightarrow x)$, analogously as above we deduce that $d_{v}(x, y) \geq d_{v}(z \rightarrow x, z \rightarrow y)$. So, $\max \left\{d_{v}(x \rightarrow z, y \rightarrow z), d_{v}(z \rightarrow x, z \rightarrow y)\right\} \leq d_{v}(x, y)$, for every $x, y, z \in A$.

(ii). First, we suppose that $d_{v}$ is a metric on $A$ and let $x \in A$ such that $v(x)=0$. Since $d_{v}(x, 1)=v(x \rightarrow 1)+v(1 \rightarrow x)=v(1)+v(x)=0+0=0$, then $x=1$, that is, $v$ is a valuation on $A$. Conversely, if $v$ is a valuation on $A$, let $x, y \in A$ such that $d_{v}(x, y)=0$. We obtain $v(x \rightarrow y)=v(y \rightarrow x)=0$. Hence $x \rightarrow y=y \rightarrow x=1$, so $x=y$, that is, $d_{v}$ is a metric on $A$.

We shall call $d_{v}$ the pseudo-metric (metric) induced by the pseudo-valuation (valuation) $v$. If we interpret a pseudo-valuation as a measure, then $d_{v}$ is well known metric induced by a measure.

Corollary 2.1. Let $v: A \rightarrow \mathbf{R}$ be a valuation. Then the operation $\rightarrow: A \times A \rightarrow A$ is uniformly continuous.

Proof. Let $x, x^{\prime}, y, y^{\prime} \in A$ and $0<\varepsilon \in \mathbf{R}$. Then $\overline{d_{v}}: A \times A \rightarrow \mathbf{R}, \overline{d_{v}}\left((x, y),\left(x^{\prime}, y^{\prime}\right)\right)=$ $\max \left\{d_{v}\left(x, x^{\prime}\right), d_{v}\left(y, y^{\prime}\right)\right\}$, for every $(x, y),\left(x^{\prime}, y^{\prime}\right) \in A \times A$ is a metric on $A \times A$. Obviously, by definition, $\overline{d_{v}}$ is a positive function. Since $v$ is a valuation on $A$, using Theorem 2.1, we deduce that $d_{v}$ is a metric on $A$. Thus, $\overline{d_{v}}((x, y),(x, y))=\max \left\{d_{v}(x, x), d_{v}(y, y)\right\}=0$ and $\overline{d_{v}}\left((x, y),\left(x^{\prime}, y^{\prime}\right)\right)=\max \left\{d_{v}\left(x, x^{\prime}\right), d_{v}\left(y, y^{\prime}\right)\right\}=\max \left\{d_{v}\left(x^{\prime}, x\right), d_{v}\left(y^{\prime}, y\right)\right\}=\overline{d_{v}}\left(\left(x^{\prime}, y^{\prime}\right),(x, y)\right)$, for every $(x, y),\left(x^{\prime}, y^{\prime}\right) \in A \times A$. Also, for $(x, y),\left(x^{\prime}, y^{\prime}\right),\left(x^{\prime \prime}, y^{\prime \prime}\right) \in A \times A$ we have: $\overline{d_{v}}\left((x, y),\left(x^{\prime \prime}, y^{\prime \prime}\right)\right)=\max \left\{d_{v}\left(x, x^{\prime \prime}\right), d_{v}\left(y, y^{\prime \prime}\right)\right\} \leq \max \left\{d_{v}\left(x, x^{\prime}\right)+d_{v}\left(x^{\prime}, x^{\prime \prime}\right), d_{v}\left(y, y^{\prime}\right)+\right.$ $\left.d_{v}\left(y^{\prime}, y^{\prime \prime}\right)\right\} \leq \max \left\{d_{v}\left(x, x^{\prime}\right), d_{v}\left(y, y^{\prime}\right)\right\}+\max \left\{d_{v}\left(x^{\prime}, x^{\prime \prime}\right), d_{v}\left(y^{\prime}, y^{\prime \prime}\right)\right\}=\overline{d_{v}}\left((x, y),\left(x^{\prime}, y^{\prime}\right)\right)+$ $\overline{d_{v}}\left(\left(x^{\prime}, y^{\prime}\right),\left(x^{\prime \prime}, y^{\prime \prime}\right)\right)$ and $\overline{d_{v}}\left((x, y),\left(x^{\prime}, y^{\prime}\right)\right)=0$ implies $d_{v}\left(x, x^{\prime}\right)=d_{v}\left(y, y^{\prime}\right)=0$ so, $x=$ $x^{\prime}$ and $y=y^{\prime}$. We conclude that $(x, y)=\left(x^{\prime}, y^{\prime}\right)$. Thus, $\overline{d_{v}}$ is a metric on $A \times A$. If $\overline{d_{v}}\left((x, y),\left(x^{\prime}, y^{\prime}\right)\right)<\varepsilon / 2$ then $d_{v}\left(x, x^{\prime}\right), d_{v}\left(y, y^{\prime}\right)<\varepsilon / 2$. We have $d_{v}\left(x \rightarrow y, x^{\prime} \rightarrow y^{\prime}\right) \leq$ $d_{v}\left(x \rightarrow y, x^{\prime} \rightarrow y\right)+d_{v}\left(x^{\prime} \rightarrow y, x^{\prime} \rightarrow y^{\prime}\right) \leq d_{v}\left(x, x^{\prime}\right)+d_{v}\left(y, y^{\prime}\right) \leq \varepsilon / 2+\varepsilon / 2=\varepsilon$, that is, $\rightarrow$ is uniformly continuous.

We have the following theorems of extension:

Theorem 2.2. Let $A$ and $B$ two BCK algebras such that $A$ is a subalgebra of $B$ and $v: A \rightarrow \mathbf{R}$ is a pseudo-valuation on $A$. Then there exists a pseudo-valuation $v^{\prime}: B \rightarrow \mathbf{R}$ such that $v_{\mid A}^{\prime}=v$.

Proof. For $x \in B$ we define $v^{\prime}(x)=\inf \left\{\sum_{i=1}^{n} v\left(x_{i}\right): x_{1}, \ldots, x_{n} \in A\right.$ and $\left(x_{1}, \ldots, x_{n} ; x\right)=$ $1\}$. Since $1 \in A$ and $1 \rightarrow 1=1$ we deduce that $v^{\prime}(1)=v(1)=0$. For $x, y \in B$, let $x_{1}, \ldots, x_{n}, z_{1}, \ldots, z_{m} \in A$ such that $\left(x_{1}, \ldots, x_{n} ; x\right)=\left(z_{1}, \ldots, z_{m} ; x \rightarrow y\right)=1$. We deduce that $\left(x_{1}, \ldots, x_{n}, z_{1}, \ldots, z_{m} ; y\right)=1$, hence, by the definition of $v^{\prime}$ we have $v^{\prime}(y) \leq \sum_{i=1}^{m} v\left(z_{i}\right)+$ $\sum_{i=1}^{n} v\left(x_{i}\right)$, so, $v^{\prime}(y) \leq \inf \left\{\sum_{i=1}^{m} v\left(z_{i}\right): z_{1}, \ldots, z_{m} \in A\right.$ and $\left.\left(z_{1}, \ldots, z_{m} ; x \rightarrow y\right)=1\right\}+\inf \left\{\sum_{i=1}^{n} v\left(x_{i}\right)\right.$ $: x_{1}, \ldots, x_{n} \in A$ and $\left.\left(x_{1}, \ldots, x_{n} ; x\right)=1\right\}$.

Thus, $v^{\prime}(y) \leq v^{\prime}(x \rightarrow y)+v^{\prime}(x)$, so, $v^{\prime}(y)-v^{\prime}(x) \leq v^{\prime}(x \rightarrow y)$, for every $x, y \in B$. We conclude that $v^{\prime}$ is a pseudo-valuation on $B$. 
If $x \in A$, since $x \rightarrow x=1$, we deduce that $v^{\prime}(x) \leq v(x)$. Let $x_{1}, \ldots, x_{n} \in A$ such that $\left(x_{1}, \ldots, x_{n} ; x\right)=1$. From Lemma 2.1, $v(x) \leq \sum_{i=1}^{n} v\left(x_{i}\right)$, hence $v(x) \leq \inf \left\{\sum_{i=1}^{n} v\left(x_{i}\right)\right.$ : $x_{1}, \ldots, x_{n} \in A$ and $\left.\left(x_{1}, \ldots, x_{n} ; x\right)=1\right\}=v^{\prime}(x)$, that is, $v_{\mid A}^{\prime}=v$.

Remark 2.3. If $A$ and $B$ are two BCK algebras such that $A$ is a subalgebra of $B, v: A \rightarrow \mathbf{R}$ is a pseudo-valuation on $A$ and $v^{\prime}: B \rightarrow \mathbf{R}$ is a real-valued function such that $v_{\mid A}^{\prime}=v$, then $v^{\prime}$ is not necessarily a pseudo-valuation on $B$. Indeed, let $B=\{0, a, b, c, 1\}$ be BCK algebra from Example 1.1. Obviously, $A=\{1\}$ is a sub-BCK-algebra of $B$ and $v: A \rightarrow \mathbf{R}$, $v(1)=0$ is a pseudo-valuation on $A$, see Example 2.2. Let $v^{\prime}: B \rightarrow \mathbf{R}$ be a real-valued function on $B$ defined by $v^{\prime}=\left(\begin{array}{ccccc}0 & a & b & c & 1 \\ 7 & 2 & 2 & 2 & 0\end{array}\right)$. Then $v_{\mid A}^{\prime}=v$, but $v^{\prime}$ is not a pseudovaluation on $B$ since $v^{\prime}(b \rightarrow 0)=v^{\prime}(a)=2<v^{\prime}(0)-v^{\prime}(b)=7-2=5$.

We consider $D \in D s(A)$ and the relation $\delta_{D}$ on $A$ defined by $(x, y) \in \delta_{D}$ iff $x \rightarrow y \in D$ and $y \rightarrow x \in D$. Hence $\delta_{D}$ is a congruence on $A$, see [3] and [13]. For $x \in A$ we denote by $x / D$ the congruence class of $x$ modulo $\delta_{D}$ and by $A / D=\{x / D: x \in A\}$ the quotient algebra. Then $A / D$ is a BCK algebra, where for $x, y \in A, x / D \rightarrow y / D=(x \rightarrow y) / D$. Also, we denote by $p_{D}: A \rightarrow A / D$ the canonical surjective morphism of BCK algebras, $p_{D}(x)=x / D$, for every $x \in A$. For $x \in D$, we have $x / D=1 / D=\mathbf{1}$.

Theorem 2.3. If $D \in D s(A)$ and $v: A \rightarrow \mathbf{R}$ is a pseudo-valuation (valuation) on $A$, then the following assertions are equivalent:

(i) There exists a pseudo-valuation (valuation) $v^{\prime}: A / D \rightarrow \mathbf{R}$ such that $v^{\prime} \circ p_{D}=v$;

(ii) $v(a)=0$, for every $a \in D$.

Proof. $(i) \Rightarrow(i i)$. Let $v^{\prime}: A / D \rightarrow \mathbf{R}$ be a pseudo-valuation on $A / D$ such that $v^{\prime} \circ p_{D}=v$ and let $a \in D$. Then $v(a)=\left(v^{\prime} \circ p_{D}\right)(a)=v^{\prime}\left(p_{D}(a)\right)=v^{\prime}(\mathbf{1})=0$.

$(i i) \Rightarrow(i)$. For $x \in A$ we define $v^{\prime}(x / D)=v(x)$. Let $x, y \in A$ such that $x / D=y / D$. Then $x \rightarrow y \in D$ and $y \rightarrow x \in D$. We obtain $0=v(x \rightarrow y) \geq v(y)-v(x)$ and $0=$ $v(y \rightarrow x) \geq v(x)-v(y)$, so, $v(x)=v(y)$, hence $v^{\prime}$ is correctly defined. Also, we have $v^{\prime}(1 / D)=v(1)=0$ and for $x, y \in A, v^{\prime}(x / D \rightarrow y / D)=v^{\prime}((x \rightarrow y) / D)=v(x \rightarrow y) \geq$ $v(y)-v(x)=v^{\prime}(y / D)-v^{\prime}(x / D)$, hence $v^{\prime}$ is a pseudo-valuation on $A$. Clearly, $v^{\prime} \circ p_{D}=v$. If $v$ is a valuation on $A$ and $x \in A$ such that $v^{\prime}(x / D)=0$, then $v(x)=0$, hence $x=1$. Thus, $x / D=1 / D=1$. We conclude that $v^{\prime}$ is a valuation on $A / D$ such that $v^{\prime} \circ p_{D}=v$.

\section{THE DUAL BCK ALGEBRA}

In this section we introduce the notion of dual BCK algebra and taking as guide line [1], we obtain results for BCK algebras.

Let $A \in D s(A), D_{1} \wedge D_{2}=D_{1} \cap D_{2}, D_{1} \vee D_{2}=<D_{1} \cup D_{2}>$ and $D_{1} \rightarrow D_{2}=\vee\{D \in$ $\left.D s(A): D_{1} \cap D \subseteq D_{2}\right\}=\left\{a \in A: D_{1} \cap<a>\subseteq D_{2}\right\}$.

Definition 3.1. The dual BCK algebra of $A$, denoted by $A^{\circ}$, is the Heyting algebra $D s(A)$ with the order $D_{1} \leq D_{2}$ iff $D_{2} \subseteq D_{1}$.

In $\left(A^{\circ}, \leq\right), \mathbf{0}=A, \mathbf{1}=\{1\}$ and for $D_{1}, D_{2} \in A^{\circ}, D_{1} \sqcap D_{2}=<D_{1} \cup D_{2}>=D_{1} \vee D_{2}$, $D_{1} \sqcup D_{2}=D_{1} \cap D_{2}$ and $D_{1} \rightarrow D_{2}=\sqcup\left\{D \in A^{\circ}: D_{1} \sqcap D \leq D_{2}\right\}=\cap\left\{D \in A^{\circ}: D_{2} \subseteq\right.$ $\left.D_{1} \vee D\right\}$.

Example 3.5. Let $A$ be the BCK algebra from Example 1.1. It is imediate to prove that

$$
D s(A)=\{\{1\},\{1, c\},\{1, a, c\},\{1, b, c\}, A\}
$$

and $A^{\circ}$ is the Heyting algebra $D s(A)$ with the order $D_{1} \leq D_{2}$ iff $D_{2} \subseteq D_{1}$, for $D_{1}, D_{2} \in$ $D s(A)$. 
Also, we define $j_{A}: A \rightarrow A^{\circ}, j_{A}(a)=<a>$, for every $a \in A$. Hence $j_{A}(1)=\mathbf{1}$ and $j_{A}(x \rightarrow y) \supseteq j_{A}(x) \rightarrow j_{A}(y)$, for every $x, y \in A$. Indeed, $j_{A}(1)=<1>=\{1\}=1$. Also, $j_{A}(x) \rightarrow j_{A}(y)=\left\langle x>\rightarrow<y>=\cap\{D \in D s(A):<y>\subseteq<x>\vee D\}\right.$. Since from $\left(c_{1}\right)$, $x \rightarrow((x \rightarrow y) \rightarrow y)=1$, we deduce (using $\left.\left(c_{6}\right)\right)$ that $y \in<x>\vee\langle x \rightarrow y>$, so $<$ $y>\subseteq<x>\vee<x \rightarrow y>$. Thus, $j_{A}(x \rightarrow y)=<x \rightarrow y>\supseteq j_{A}(x) \rightarrow j_{A}(y)$, for every $x, y \in A$.

Lemma 3.3. For every $x, y \in A$, there is a natural number $m \geq 1$ such that

$$
j_{A}\left(x \rightarrow_{m} y\right) \subseteq j_{A}(x) \rightarrow j_{A}(y) .
$$

Proof. We have that $j_{A}(x) \rightarrow j_{A}(y)=\cap\{D \in D s(A):<y>\subseteq<x>\vee D\}$. So let $D \in$ $D s(A)$ such that $\langle y\rangle \subseteq<x>\vee D$. Since $\langle x>\vee D=\{z \in A: t \rightarrow(d \rightarrow z)=1$, for some $d \in D$ and $t \in\langle x\rangle\}$ and $y \in\langle y\rangle \subseteq\langle x\rangle \vee D$, we deduce that $t \rightarrow(d \rightarrow y)=1$, for some $d \in D$ and $t \in\langle x\rangle$. But $t \rightarrow(d \rightarrow y)=d \rightarrow(t \rightarrow y)$, so, $d \rightarrow(t \rightarrow y)=1$ and $d \leq t \rightarrow y$. We deduce that $t \rightarrow y \in D$ for some $t \in<x>$ (i.e., $x \rightarrow_{n} t=1$, for some $n \geq 1)$.

Finally, $x \rightarrow_{n} y \in D$, for some $n \geq 1$. Hence, there is a natural number $m \geq 1$ such that $x \rightarrow_{m} y \in D$, for every $D \in D s(A)$. We conclude that there is $m \geq 1$ such that $j_{A}\left(x \rightarrow_{m} y\right) \subseteq j_{A}(x) \rightarrow j_{A}(y)$, for every $x, y \in A$.

We recall that if $A$ and $B$ are two BCK algebras, a function $f: A \rightarrow B$ is a morphism of BCK algebras if $f(x \rightarrow y)=f(x) \rightarrow f(y)$ for every $x, y \in A$.

Lemma 3.4. $j_{B(A)}$ is an injective morphism of BCK algebras.

Proof. We recall that (see [6]) if $a \in B(A)$ then $a \rightarrow(a \rightarrow x)=a \rightarrow x$ for every $x \in A$, so, $\langle a\rangle=\{x \in A: a \leq x\}$. Using Lemma 3.3, if we consider $x, y \in B(A)$ we deduce that $j_{A}(x) \rightarrow j_{A}(y)=j_{A}(x \rightarrow y)$. Also, if $j_{A}(x)=j_{A}(y)$, then $\langle x\rangle=<y>$ so, $x \leq y$ and $y \leq x$. Thus, $x=y$. We conclude that $j_{B(A)}$ is an injective morphism of BCK algebras.

Definition 3.2. We say that a BCK algebra $A$ has property $\mathcal{F}$ if for every $D \in A^{\circ}$ there exist $x_{1}, \ldots, x_{n} \in A$ such that $D \subseteq<x_{1}, \ldots, x_{n}>$.

Example 3.6. If we consider BCK algebra from Example 1.1, then $A$ has property $\mathcal{F}$ since $A^{\circ}=D s(A)=\{\{1\},\{1, c\},\{1, a, c\},\{1, b, c\}, A\}$ and $\{1\}=<1>,\{1, c\}=<1, c>$, $\{1, a, c\}=<1, a, c>,\{1, b, c\}=<1, b, c>$ and $A=<0>$.

Remark 3.4. Examples of BCK algebras with property $\mathcal{F}$ are bounded BCK algebras (since $A=<0>$ ) and finite BCK algebras.

Theorem 3.4. Let $A$ be a BCK algebra with property $\mathcal{F}$ and $v: A \rightarrow \mathbf{R}$ a pseudo-valuation on $A$. Then there exists a pseudo-valuation on $A^{\circ}, v^{\prime}: A^{\circ} \rightarrow \mathbf{R}$ such that $v^{\prime} \circ j_{A}=v$.

Proof. For $D \in A^{\circ}$ we define $v^{\prime}(D)=\inf \left\{\sum_{i=1}^{n} v\left(x_{i}\right): x_{1}, \ldots, x_{n} \in A\right.$ and $\left.D \subseteq<x_{1}, \ldots, x_{n}>\right\}$. First, we prove that $v /$ is a pseudo-valuation on $A^{\circ}$.

Clearly, $v^{\prime}(\mathbf{1})=\inf \left\{\sum_{i=1}^{n} v\left(x_{i}\right): x_{1}, \ldots, x_{n} \in A\right.$ and $\left.\{1\} \subseteq<x_{1}, \ldots, x_{n}>\right\}=v(1)=0$. To verify $\left({ }^{*}\right)$, let $D_{1}, D_{2} \in A^{\circ}$ and $x_{1}, \ldots, x_{n}, z_{1}, \ldots, z_{m} \in A$ such that $D_{1} \subseteq<x_{1}, \ldots, x_{n}>$ and $D_{1} \rightarrow D_{2} \subseteq<z_{1}, \ldots, z_{m}>$. Then $D_{2} \subseteq D_{1} \vee\left(D_{1} \rightarrow D_{2}\right) \subseteq<x_{1}, \ldots, x_{n}>\vee<z_{1}, \ldots, z_{m}>$ $\subseteq<x_{1}, \ldots, x_{n}, z_{1}, \ldots, z_{m}>$. Thus $v^{\prime}\left(D_{2}\right) \leq \sum_{i=1}^{n} v\left(x_{i}\right)+\sum_{j=1}^{m} v\left(z_{j}\right)$, so, $v^{\prime}\left(D_{2}\right) \leq \inf \left\{\sum_{i=1}^{n} v\left(x_{i}\right)\right.$ : $x_{1}, \ldots, x_{n} \in A$ and $\left.D_{1} \subseteq<x_{1}, \ldots, x_{n}>\right\}+\inf \left\{\sum_{j=1}^{m} v\left(z_{j}\right): z_{1}, \ldots, z_{m} \in A\right.$ and $D_{1} \rightarrow D_{2} \subseteq<$ $\left.z_{1}, \ldots, z_{m}>\right\}=$ 
$v^{\prime}\left(D_{1}\right)+v^{\prime}\left(D_{1} \rightarrow D_{2}\right)$. We obtain that $v^{\prime}\left(D_{1} \rightarrow D_{2}\right) \geq v^{\prime}\left(D_{2}\right)-v^{\prime}\left(D_{1}\right)$.

If $a, x_{1}, \ldots, x_{n} \in A$ such that $\langle a\rangle \subseteq<x_{1}, \ldots, x_{n}>$ then $\left(x_{i_{1}}, \ldots, x_{i_{k}} ; a\right)=1$, for some $x_{i_{1}}, \ldots, x_{i_{k}} \in\left\{x_{1}, \ldots, x_{n}\right\}$. From Lemma 2.1 we deduce that $v(a) \leq \sum_{i=1}^{n} v\left(x_{i}\right)$, so $v(a) \leq$ $\inf \left\{\sum_{i=1}^{n} v\left(x_{i}\right): x_{1}, \ldots, x_{n} \in A\right.$ and $\left.<a>\subseteq<x_{1}, \ldots, x_{n}>\right\}=v^{\prime}(<a>)$. Since $<a>\subseteq<$ $\{a\}>$ it follows that $v^{\prime}(<a>)=v(a)$. We conclude that $v^{\prime} \circ j_{A}=v$.

\title{
4. CONCLUSIONS AND FUTURE WORK
}

In [1], is defined a pseudo-valuation on a Hilbert algebra. In this paper, we generalize this concept for BCK algebras and we prove theorems on extensions of pseudo-valuations (valuations) on BCK algebras. Since the power set of a non-empty set is a BCK algebra, using of pseudo-valuations can be useful in the study of theory of sets. As another direction of research one could define and study the concept of free Hilbert algebra with infimum over a BCK algebra. Specifically, the questions are the following: Are there the free Hilbert algebras with infimum over BCK algebras? and if the answer is positive, Which is the relation of these algebras with pseudo-valuations? It is interesting to note that, if the BCK algebra is a Hilbert algebra, then an explicit construction of the free semilattice extension of a Hilbert algebra is not immediate. Also, [1] contains results about pseudo-valuations on free Hertz algebra over a Hilbert algebra.

\section{REFERENCES}

[1] Busneag, D. On extensions of pseudo-valuations on Hilbert algebras. Discrete Mathematics 263 (2003), 11-24.

[2] Celani, S. Deductive systems of BCK-algebras. Acta Univ. Palack. Olomuc. Fac. Rerum Natur. Math. 43 (2004), 27-32.

[3] Ciungu, L. Non-commutative Multiple-Valued Logic Algebras, Springer, 2013.

[4] Doh, M. I.; Kang, M. S. BCI / BCK-algebras with pseudo-valuations. Honam Math. J. 32 (2010), no. 2, 217-226.

[5] Doh, M. I.; Kang, M. S. Commutative pseudo-valuations on BCK-algebras. Int. J. Math. Sci. (2011), Art. Id. 754047, 6 pp.

[6] Gispert, J.; Torrens, A. Boolean representation of Bounded BCK-algebras. Soft Computing 12 (2008), no. 10, 941-954.

[7] Ghorbani, S. Quotient BCI-algebras induced by pseudo-valuations. Iran. J. Math. Sci. Inform. 5 (2010), no. 2, 13-24.

[8] Iorgulescu, A. Algebras of logic as BCK algebras. ASE Ed., Bucharest, 2008.

[9] Isèki, K. An algebra related with a propositional calculus. Proc. Jpn. Acad. 42 (1966), 26-29.

[10] Isèki, K.; Tanaka, S. An introduction to the theory of BCK - algebras. Math. Japonica 23 (1978), no. 1, 1-26.

[11] Jun, Y. B.; Lee, J. K. ; Ahn, S. S. Positive implicative pseudo-valuations on BCK-algebras. Appl. Math. Sci. (Ruse) 5 (2011), no. 13-16, 651-662.

[12] Kouhestani, N.; Mehrshad, S. (Semi) topological quotient BCK-algebras. Afr. Mat. 28 (2017), no. 7-8, 1235-1251.

[13] Kühr, J. Pseudo-BCK-algebras and related structures. Univ. Palackeho v Olomuci, 2007.

[14] Mehrshad, S.; Kouhestani, N. A quasi-uniformity on BCC-algebras. An. Univ. Craiova, Ser. Mat. Inform. 44 (2017), no. 1, 64-77.

[15] Mehrshad, S.; Kouhestani, N. On pseudo-valuations on BCK-algebras. Filomat 32 (2018), no. 12, 4319-4332.

[16] Senapati, T.; Shum, K. P. Cubic implicative ideals of BCK-algebras. Missouri J. Math. Sci. 29 (2017), no. 2, 125-138.

\author{
DEPARTMENT OF MATHEMATICS \\ UNIVERSITY OF CRAIOVA \\ Al. I. CUZA 13, 200585 CRAIOVA, RomAniA \\ Email address: busneag@ central .ucv. ro \\ Email address: piciudanamarina@yahoo.com \\ Email address: istratamihaela@yahoo.com
}

